\title{
Research Article \\ On Some Arithmetical Properties of the Genocchi Numbers and Polynomials
}

\author{
Kyoung Ho Park ${ }^{1}$ and Young-Hee Kim² \\ ${ }^{1}$ Department of Mathematics, Kyungpook National University, Daegu 702-701, South Korea \\ ${ }^{2}$ Division of General Education-Mathematics, Kwangwoon University, Seoul 139-701, South Korea \\ Correspondence should be addressed to Young-Hee Kim, yhkim@kw.ac.kr
}

Received 31 October 2008; Revised 19 December 2008; Accepted 25 December 2008

Recommended by Martin J. Bohner

We investigate the properties of the Genocchi functions and the Genocchi polynomials. We obtain the Fourier transform on the Genocchi function. We have the generating function of $(h, q)$-Genocchi polynomials. We define the Cangul-Ozden-Simsek's type twisted $(h, q)$-Genocchi polynomials and numbers. We also have the generalized twisted $(h, q)$-Genocchi numbers attached to the Dirichlet's character $x$. Finally, we define zeta functions related to $(h, q)$-Genocchi polynomials and have the generating function of the generalized $(h, q)$-Genocchi numbers attached to $x$.

Copyright (C) 2008 K. H. Park and Y.-H. Kim. This is an open access article distributed under the Creative Commons Attribution License, which permits unrestricted use, distribution, and reproduction in any medium, provided the original work is properly cited.

\section{Introduction}

After Carlitz introduced an interesting $q$-analogue of Frobenius-Euler numbers in [1], $q$ Bernoulli and $q$-Euler numbers and polynomials have been studied by several authors. Recently, many authors have an interest in the q-extension of the Genocchi numbers and polynomials(cf. [2-5]). Kim et al. [5] defined the $q$-Genocchi numbers and the $q$-Genocchi polynomials. In [3], Kim derived the $q$-analogs of the Genocchi numbers and polynomials by constructing $q$-Euler numbers. He also gave some interesting relations between $q$-Euler and $q$-Genocchi numbers. The first author et al. [6] obtained the distribution relation for the Genocchi polynomials.

The main aim of this paper is to derive the Fourier transform for the Genocchi function. Recently, Kim [7] investigated the properties of the Euler functions and derived the interesting formula related to the infinite series by using the Fourier transform for the Euler function. In this paper, we investigate some arithmetical properties of the Genocchi functions and the Genocchi polynomials.

In [8], Cangul-Ozden-Simsek constructed new generating functions of the twisted $(h, q)$-extension of twisted Euler polynomials and numbers attached to the Dirichlet 
character $x$. Cangul et al. [8] also defined the twisted $(h, q)$-extension of zeta functions, which interpolate the twisted $(h, q)$-extension of Euler numbers at negative integers. In this paper, we define the Cangul-Ozden-Simsek type twisted $(h, q)$-Genocchi polynomials and numbers. We have the generating function of $(h, q)$-Genocchi polynomials. We have the generalized twisted $(h, q)$-Genocchi numbers attached to the Dirichlet character $x$. We define zeta functions related to $(h, q)$-Genocchi polynomials and we have the generating function of the generalized $(h, q)$-Genocchi numbers attached to $x$.

Let $p$ be a fixed odd prime number. Throughout this paper $\mathbb{Z}_{p}, \mathbb{Q}_{p}, \mathbb{C}$, and $\mathbb{C}_{p}$ will, respectively, denote the ring of $p$-adic rational integers, the field of $p$-adic rational numbers, the complex number field, and the completion of algebraic closure of $\mathbb{Q}_{p}$. Let $\mathbb{N}$ be the set of natural numbers and $\mathbb{Z}_{+}=\mathbb{N} \cup\{0\}$. Let $v_{p}$ be the normalized exponential valuation of $\mathbb{C}_{p}$ with $|p|_{p}=p^{-v_{p}(p)}=1 / p$. When one talks of $q$-extension, $q$ is variously considered as an indeterminate, a complex number $q \in \mathbb{C}$, or a $p$-adic number $q \in \mathbb{C}_{p}$. If $q \in \mathbb{C}$, one normally assumes $|q|<1$. If $q \in \mathbb{C}_{p}$, then we assume $|1-q|_{p}<1$. We also use the following notations:

$$
[x]_{q}=[x: q]=\frac{1-q^{x}}{1-q}, \quad[x]_{-q}=\frac{1-(-q)^{x}}{1+q} .
$$

For $d$, a fixed positive integer with $(p, d)=1$, set

$$
\begin{gathered}
X=X_{d}=\frac{\lim _{N} \mathbb{Z}}{d p^{N} \mathbb{Z}}, \quad X_{1}=\mathbb{Z}_{p}, \\
X^{*}=\bigcup_{\substack{0<a<d p,(a, p)=1}}\left(a+d p \mathbb{Z}_{p}\right), \\
a+d p^{N} \mathbb{Z}_{p}=\left\{x \in X \mid x \equiv a\left(\bmod d p^{N}\right)\right\},
\end{gathered}
$$

where $a \in \mathbb{Z}$ satisfies the condition $0 \leq a<d p^{N}$. The distribution is defined by

$$
\mu_{q}\left(a+d p^{N} \mathbb{Z}_{p}\right)=\frac{q^{a}}{\left[d p^{N}\right]_{q}} .
$$

We say that $f$ is uniformly differential function at a point $a \in \mathbb{Z}_{p}$, and we write $f \in$ $U D\left(\mathbb{Z}_{p}\right)$, if the difference quotients, $F_{f}(x, y)=f(x)-f(y) / x-y$ have a limit $f^{\prime}(a)$ as $(x, y) \rightarrow$ $(a, a)$.

For $f \in U D\left(\mathbb{Z}_{p}\right)$, the $p$-adic invariant $q$-integral on $\mathbb{Z}_{p}$ is defined as

$$
I_{q}(f)=\int_{\mathbb{Z}_{p}} f(x) d \mu_{q}(x)=\lim _{N \rightarrow \infty} \frac{1}{\left[p^{N}\right]_{q}} \sum_{x=0}^{p^{N}-1} f(x) q^{x} .
$$


The fermionic $p$-adic $q$-measures on $\mathbb{Z}_{p}$ are defined as

$$
\mu_{-q}\left(a+d p^{N} \mathbb{Z}_{p}\right)=\frac{(-q)^{a}}{\left[d p^{N}\right]_{-q}}
$$

and the fermionic $p$-adic invariant $q$-integral on $\mathbb{Z}_{p}$ is defined as

$$
I_{-q}(f)=\int_{\mathbb{Z}_{p}} f(x) d \mu_{-q}(x)=\lim _{N \rightarrow \infty} \frac{1}{\left[p^{N}\right]_{-q}} \sum_{x=0}^{p^{N}-1} f(x)(-q)^{x},
$$

for $f \in U D\left(\mathbb{Z}_{p}\right)$. For $f \in U D\left(\mathbb{Z}_{p}\right)$, we note that

$$
I_{-1}\left(f_{1}\right)+I_{-1}(f)=2 f(0),
$$

where $f_{1}(x)=f(x+1)$. (For details see [1-44].)

In this paper, we investigate arithmetical properties of the Genocchi functions and the Genocchi polynomials. In Section 2, we derive the Fourier transform on the Genocchi function. In Section 3, we define the Cangul-Ozden-Simsek type twisted $(h, q)$-Genocchi polynomials and numbers. We have the generating function of $(h, q)$-Genocchi polynomials. We also have the generalized twisted $(h, q)$-Genocchi numbers attached to $x$. In Section 4 , we define zeta functions related to $(h, q)$-Genocchi polynomials and we have the generating function of the generalized $(h, q)$-Genocchi numbers attached to $x$.

\section{Genocchi numbers and functions}

The Genocchi numbers are defined as

$$
\frac{2 t}{e^{t}+1}=e^{G t}=\sum_{n=0}^{\infty} G_{n} \frac{t^{n}}{n !} \quad \text { for }|t|<\pi
$$

where we use the technique method notation by replacing $G^{n}$ by $G_{n}$, symbolically. From this definition, we can derive the following relation:

$$
G_{0}=0, \quad(G+1)^{n}+G_{n}= \begin{cases}2 & \text { if } n=1 \\ 0 & \text { if } n>1\end{cases}
$$

From (2.2), we note that $G_{0}=0, G_{1}=1, G_{2}=-1, \ldots$, and $G_{2 k+1}=0, G_{2 k} \in \mathbb{Z}(k=1,2, \ldots)$. The Genocchi polynomials $G_{n}(x)$ are defined as

$$
\frac{2 t}{e^{t}+1} e^{x t}=\sum_{n=0}^{\infty} G_{n}(x) \frac{t^{n}}{n !}, \quad \text { for } x \in \mathbb{R}
$$


From (2.1) and (2.3), we can derive

$$
G_{n}(x)=\sum_{\ell=0}^{n}\left(\begin{array}{l}
n \\
\ell
\end{array}\right) G_{\ell} x^{n-\ell}, \quad \text { where }\left(\begin{array}{l}
n \\
\ell
\end{array}\right)=\frac{n(n-1) \cdots(n-\ell+1)}{\ell !} .
$$

By (2.1), it is not difficult to show that the recurrence relation for the Genocchi numbers is given by

$$
G_{0}=0, \quad \sum_{\ell=0}^{n}\left(\begin{array}{l}
n \\
\ell
\end{array}\right) G_{\ell}+G_{n}=2 \delta_{1, n}
$$

where $\delta_{1, n}$ is the Kronecker symbol.

From (2.4) and (2.5), we note that

$$
G_{n}(1)+G_{n}=0 \quad \text { for } n \geq 2 \text {. }
$$

Thus, we obtain the following lemma.

Lemma 2.1. For $n(\geq 2) \in \mathbb{N}$, one has $G_{n}(1)=-G_{n}$.

From (2.4), we can easily derive

$$
\begin{aligned}
\frac{d}{d x} G_{n}(x) & =\frac{d}{d x} \sum_{\ell=0}^{n}\left(\begin{array}{l}
n \\
\ell
\end{array}\right) G_{\ell} x^{n-\ell}=\sum_{\ell=0}^{n}\left(\begin{array}{l}
n \\
\ell
\end{array}\right)(n-\ell) G_{\ell} x^{n-\ell-1} \\
& =n \sum_{\ell=0}^{n} \frac{(n-1) !}{(n-\ell-1) ! \ell !} G_{\ell} x^{n-1-\ell}=n \sum_{\ell=0}^{n}\left(\begin{array}{c}
n-1 \\
\ell
\end{array}\right) G_{\ell} x^{n-1-\ell}=n G_{n-1}(x) .
\end{aligned}
$$

By (2.7), we obtain the following proposition.

Proposition 2.2. For $n \geq 0$, one has

$$
\int_{0}^{x} G_{n}(t) d t=\frac{1}{n+1} G_{n+1}(x) .
$$

From now on, we assume that $G_{n}(x)$ is the Genocchi function. Let us consider the Fourier transform for the Genocchi function $G_{n}(x)$ as follows.

For $m \in \mathbb{N}$, the Fourier transform on the Genocchi function is given by

$$
G_{m}(x)=\sum_{n=-\infty}^{\infty} a_{n}^{(m)} e^{(2 n+1) \pi i x}, \quad\left(a_{n}^{(m)} \in \mathbb{C}\right),
$$

where

$$
a_{n}^{(m)}=\int_{0}^{1} G_{m}(x) e^{-(2 n+1) \pi i x} d x
$$


From (2.8) and (2.10), we note that

$$
\begin{aligned}
a_{n}^{(m)} & =\int_{0}^{1} G_{m}(x) e^{-(2 n+1) \pi i x} d x \\
& =\left[\frac{G_{m+1}(x)}{m+1} e^{-(2 n+1) \pi i x}\right]_{0}^{1}+\frac{(2 n+1) \pi i}{m+1} \int_{0}^{1} G_{m+1}(x) e^{-(2 n+1) \pi i x} d x \\
& =\frac{(2 n+1) \pi i}{m+1} a_{n}^{(m+1)}
\end{aligned}
$$

Thus, for $m \geq 2$, we have

$$
a_{n}^{(m)}=\frac{m}{(2 n+1) \pi i} a_{n}^{(m-1)}=\frac{m(m-1)}{((2 n+1) \pi i)^{2}} a_{n}^{(m-2)}=\cdots=\frac{m !}{((2 n+1) \pi i)^{m-1}} a_{n}^{(1)} .
$$

From (2.4) and (2.10), we derive

$$
a_{n}^{(1)}=\int_{0}^{1} G_{1}(x) e^{-(2 n+1) \pi i x} d x=\int_{0}^{1} e^{-(2 n+1) \pi i x} d x=\left[\frac{-e^{-(2 n+1) \pi i x}}{(2 n+1) \pi i}\right]_{0}^{1}=\frac{2}{(2 n+1) \pi i}
$$

From (2.12) and (2.13), we can derive

$$
a_{n}^{(m)}=\frac{m ! 2}{((2 n+1) \pi i)^{m}} \quad(m \in \mathbb{N}), a_{n}^{(0)}=0 .
$$

By (2.9) and (2.14), we have that $G_{0}(x)=0$ and

$$
G_{m}(x)=m ! 2 \sum_{n=-\infty}^{\infty} \frac{e^{(2 n+1) \pi i x}}{((2 n+1) \pi i)^{m}}, \quad \text { for } 0 \leq x<1
$$

Therefore, we obtain the following theorem.

Theorem 2.3. For $m \in \mathbb{Z}_{+}, x \in \mathbb{R}$ with $0 \leq x<1$, one has

$$
G_{m}(x)=m ! 2 \sum_{n=-\infty}^{\infty} \frac{e^{(2 n+1) \pi i x}}{((2 n+1) \pi i)^{m}} .
$$

If we take $x=1$, then we have

$$
G_{m}(1)=-m ! 2 \sum_{n=-\infty}^{\infty} \frac{1}{((2 n+1) \pi i)^{m}}
$$

By (2.17) and Lemma 2.1, we obtain the following corollary. 
Corollary 2.4. For $m \in \mathbb{Z}_{+}$, one has

$$
G_{m}=m ! 2 \sum_{n=-\infty}^{\infty} \frac{1}{((2 n+1) \pi i)^{m}}
$$

From Corollary 2.4, we note that

$$
G_{2 m}=(2 m) ! 2 \sum_{n=-\infty}^{\infty} \frac{1}{((2 n+1) \pi i)^{2 m}}=(2 m) ! 2 \sum_{n=-\infty}^{\infty} \frac{1}{(2 n+1)^{2 m} \pi^{2 m}(-1)^{m}}
$$

Thus, we have

$$
\sum_{n=-\infty}^{\infty} \frac{1}{(2 n+1)^{2 m}}=(-1)^{m} \frac{G_{2 m}}{2(2 m) !} \pi^{2 m}
$$

By (2.20), we obtain the following corollary.

Corollary 2.5. For $m \in \mathbb{Z}_{+}$, one has

$$
\sum_{n=1}^{\infty} \frac{1}{(2 n+1)^{2 m}}=(-1)^{m} \frac{G_{2 m}}{4(2 m) !} \pi^{2 m}
$$

\section{3. $(h, q)$-extension of twisted Genocchi numbers and polynomials}

In this section, we will define the $(h, q)$-extensions of twisted Genocchi numbers and polynomials which are the Cangul-Ozden-Simsek type twisted $(h, q)$-Genocchi numbers and polynomials, respectively. We will have the generating function of $(h, q)$-Genocchi polynomials and the generalized twisted $(h, q)$-Genocchi numbers attached to $x$.

Let $f(x)=e^{x t}$. Then, we have from the definition of the Genocchi numbers and the fermionic $p$-adic $q$-integral on $\mathbb{Z}_{p}$ that

$$
\begin{gathered}
t \int_{\mathbb{Z}_{p}} e^{x t} d \mu_{-1}(x)=\sum_{n=0}^{\infty} G_{n} \frac{t^{n}}{n !}=t \sum_{n=0}^{\infty} \frac{G_{n+1}}{n+1} \frac{t^{n}}{n !}, \\
t \int_{\mathbb{Z}_{p}} e^{(x+y) t} d \mu_{-1}(y)=\sum_{n=0}^{\infty} G_{n}(x) \frac{t^{n}}{n !}=t \sum_{n=0}^{\infty} \frac{G_{n+1}(x)}{n+1} \frac{t^{n}}{n !} .
\end{gathered}
$$

Thus, we obtain

$$
\int_{\mathbb{Z}_{p}} x^{n} d \mu_{-1}(x)=\frac{G_{n+1}}{n+1}, \quad \int_{\mathbb{Z}_{p}}(x+y)^{n} d \mu_{-1}(y)=\frac{G_{n+1}(x)}{n+1} .
$$


For $f \in U D\left(\mathbb{Z}_{p}\right)$ and $n \in \mathbb{N}$, we have

$$
\int_{\mathbb{Z}_{p}} f(x+n) d \mu_{-1}(x)=(-1)^{n} \int_{\mathbb{Z}_{p}} f(x) d \mu_{-1}(x)+2 \sum_{\ell=0}^{n-1}(-1)^{n-1+\ell} f(\ell) .
$$

By (3.2) and (3.3), if we take $f(x)=x^{k}\left(k \in \mathbb{Z}^{+}\right)$, we easily see that

$$
\int_{\mathbb{Z}_{p}}(x+n)^{k} d \mu_{-1}(x)-\int_{\mathbb{Z}_{p}} x^{k} d \mu_{-1}(x)=2 \sum_{\ell=0}^{n-1}(-1)^{\ell-1} \ell^{k} \quad \text { if } n \equiv 0(\bmod 2) .
$$

Thus, we have

$$
\frac{G_{k+1}(n)}{k+1}-\frac{G_{k+1}}{k+1}=2 \sum_{\ell=0}^{n-1}(-1)^{\ell-1} \ell^{k} \quad \text { if } n \equiv 0(\bmod 2)
$$

If $n \equiv 1(\bmod 2)$, then we know that

$$
\int_{\mathbb{Z}_{p}}(x+n)^{k} d \mu_{-1}(x)+\int_{\mathbb{Z}_{p}} x^{k} d \mu_{-1}(x)=2 \sum_{\ell=0}^{n-1}(-1)^{\ell} \ell^{k} .
$$

Thus, we get

$$
\frac{G_{k+1}(n)}{k+1}+\frac{G_{k+1}}{k+1}=2 \sum_{\ell=0}^{n-1}(-1)^{\ell} \ell^{k} \quad \text { if } n \equiv 1(\bmod 2)
$$

We can consider the generalized Genocchi numbers as follows:

$$
\frac{G_{n+1}}{n+1}=\int_{X} x^{n} d \mu_{-1}(x), \quad G_{0}=0
$$

where $n \in \mathbb{Z}_{+}$. Let $d \in \mathbb{N}$ with $d \equiv 1(\bmod 2)$. From (3.3) and (3.8), we note that

$$
t \int_{X} e^{x t} d \mu_{-1}(x)=\frac{2 \sum_{\ell=0}^{d-1}(-1)^{\ell} e^{\ell t}}{e^{d t}+1} t=\sum_{n=0}^{\infty} \frac{G_{n}}{n !} t^{n}
$$


By (3.8) and (3.9), it is not difficult to show that

$$
\begin{aligned}
\frac{G_{n+1}}{n+1} & =\int_{X} x^{n} d \mu_{-1}(x) \\
& =d^{n} \sum_{a=0}^{d-1}(-1)^{a} \int_{\mathbb{Z}_{p}}\left(\frac{a}{d}+x\right)^{n} d \mu_{-1}(x) \\
& =d^{n} \sum_{a=0}^{d-1}(-1)^{a} \frac{G_{n+1}(a / d)}{n+1} \\
\int_{X}(x+y)^{n} d \mu_{-1}(y) & =d^{n} \sum_{a=0}^{d-1}(-1)^{a} \int_{\mathbb{Z}_{p}}\left(\frac{x+a}{d}+y\right)^{n} d \mu_{-1}(y) .
\end{aligned}
$$

Thus, the distribution relations for the Genocchi numbers and the Genocchi polynomials for $d \in \mathbb{N}$ with $d \equiv 1(\bmod 2)$ are obtained as follows (cf. [6]):

$$
\begin{gathered}
\frac{G_{n+1}}{n+1}=d^{n} \sum_{a=0}^{d-1}(-1)^{a} \frac{G_{n+1}(a / d)}{n+1}, \\
\frac{G_{n+1}(x)}{n+1}=d^{n} \sum_{a=0}^{d-1}(-1)^{a} \frac{G_{n+1}((x+a) / d)}{n+1} .
\end{gathered}
$$

By using the multivariate integral, we can also consider the multiple Genocchi numbers and polynomials.

Let $q \in \mathbb{C}_{p}$ with $|1-q|_{p}<1$ be indeterminate and let $h \in \mathbb{Z}$. Then, we note that

$$
t \int_{\mathbb{Z}_{p}} q^{h y} e^{(x+y) t} d \mu_{-1}(y)=\frac{2 t}{q^{h} e^{t}+1} e^{x t} .
$$
as follows:

Now, we define the Cangul-Ozden-Simsek type $(h, q)$-Genocchi polynomials $G_{n, q}^{(h)}(x)$

$$
\frac{2 t}{q^{h} e^{t}+1} e^{x t}=\sum_{n=0}^{\infty} G_{n, q}^{(h)}(x) \frac{t^{n}}{n !} .
$$

From (3.13), we note that

$$
\int_{\mathbb{Z}_{p}} q^{h y}(x+y)^{n} d \mu_{-1}(y)=\frac{G_{n+1, q}^{(h)}(x)}{n+1} .
$$

Let $C_{p^{n}}$ be the space of primitive $p^{n}$-th root of unity with

$$
C_{p^{n}}=\left\{\xi \in \mathbb{C}_{p} \mid \xi^{p^{n}}=1\right\},
$$


and let $T_{p}$ be the direct limit of $C_{p^{n}}$, that is,

$$
T_{p}=\lim _{n \rightarrow \infty} C_{p^{n}}=\bigcup_{n \geq 0} C_{p^{n}}
$$

and then $T_{p}$ is a $p$-adic locally constant space.

For $\xi \in T_{p}$, we define the Cangul-Ozden-Simsek type twisted $(h, q)$-Genocchi polynomials $G_{n, q \cdot \xi}^{(h)}(x)$ as follows:

$$
t \int_{\mathbb{Z}_{p}} q^{h y} \xi^{y} e^{(x+y) t} d \mu_{-1}(y)=\frac{2 t}{\xi q^{h} e^{t}+1} e^{x t}=\sum_{n=0}^{\infty} G_{n, q, \xi}^{(h)}(x) \frac{t^{n}}{n !} .
$$

By (3.17), we have

$$
\int_{\mathbb{Z}_{p}} q^{h y} \xi^{y}(x+y)^{n} d \mu_{-1}(y)=\frac{G_{n+1, q, \xi}^{(h)}(x)}{n+1} .
$$

From the result of Cangul et al. [8], we note that

$$
E_{n+1, q, \xi}^{(h)}(x)=\frac{G_{n+1, q, \xi}^{(h)}(x)}{n+1},
$$

where $E_{n+1, q, \xi}^{(h)}(x)$ is the twisted $(h, q)$-Euler polynomials.

Let $x$ be the Dirichlet character with conductor $d \in \mathbb{N}$ with $d \equiv 1(\bmod 2)$. Then, we consider the generalized Genocchi numbers attached to $x$ as follows:

$$
\frac{G_{n+1, x}}{n+1}=\int_{X} x(x) x^{n} d \mu_{-1}(x), \quad G_{0, x}=0,
$$

where $n \in \mathbb{Z}_{+}$.

From (3.3) and (3.20), we note that

$$
t \int_{X} e^{x t} X(x) d \mu_{-1}(x)=\frac{2 \sum_{\ell=0}^{d-1}(-1)^{\ell} X(\ell) e^{\ell t}}{e^{d t}+1} t=\sum_{n=0}^{\infty} \frac{G_{n, x}}{n !} t^{n}
$$


By (3.20) and (3.21), it is not difficult to show that

$$
\begin{aligned}
\frac{G_{n+1, x}}{n+1} & =\int_{X} x(x) x^{n} d \mu_{-1}(x) \\
& =d^{n} \sum_{a=0}^{d-1}(-1)^{a} x(a) \int_{\mathbb{Z}_{p}}\left(\frac{a}{d}+x\right)^{n} d \mu_{-1}(x) \\
& =d^{n} \sum_{a=0}^{d-1}(-1)^{a} \chi(a) \frac{G_{n+1}(a / d)}{n+1}
\end{aligned}
$$

Now, we also consider the Cangul-Ozden-Simsek type twisted $(h, q)$-Genocchi numbers attached to $X$ as follows.

For $\xi \in T_{p}$ and $h \in \mathbb{Z}$, we have

$$
\begin{aligned}
t \int_{X} X(x) \xi^{x} q^{h x} e^{x t} d \mu_{-1}(x) & =t \sum_{a=0}^{d-1}(-1)^{a} X(a) e^{a t} \xi^{a} q^{h a} \int_{\mathbb{Z}_{p}} e^{x d t} \xi^{d x} q^{h d x} d \mu_{-1}(x) \\
& =\frac{2 t \sum_{a=0}^{d-1}(-1)^{a} X(a) e^{a t} \xi^{a} q^{h a}}{q^{h d} \xi^{d} e^{d t}+1} \\
& =\sum_{n=0}^{\infty} G_{n, q, \xi, x}^{(h)} \frac{t^{n}}{n !} .
\end{aligned}
$$

From (3.23), we have

$$
\int_{X} X(x) \xi^{x} q^{h x} x^{n} d \mu_{-1}(x)=\frac{G_{n+1, q, \xi, x}^{(h)}}{n+1}, \quad \text { for } n \geq 0 .
$$

From the result of Cangul et al. [8], we note that

$$
E_{n, q, \xi, x}^{(h)}=\frac{G_{n+1, q, \xi, x}^{(h)}}{n+1}, \text { for } n \geq 0
$$

where $E_{n, q, \xi, x}^{(h)}$ are called the generalized twisted $(h, q)$-Euler numbers attached to $x$.

\section{Zeta functions related to the Genocchi polynomials}

In this section, we assume that $q \in \mathbb{C}$ with $|q|<1$. Let $F(t, x)$ be the generating function of $(h, q)$-Genocchi polynomials defined as follows:

$$
F(t, x)=\frac{2 t}{q^{h} e^{t}+1} e^{x t}=\sum_{n=0}^{\infty} G_{n, q}^{(h)}(x) \frac{t^{n}}{n !}
$$

where $|t+h \log q|<\pi$. 
K. H. Park and Y.-H. Kim

Then, we note that

$$
F(t, x)=2 t \sum_{n=0}^{\infty}(-1)^{n} q^{n h} e^{(n+x) t}
$$

By (4.1) and (4.2), we easily see that

$$
G_{k, q}^{(h)}(x)=\left.\frac{d^{k}}{d t^{k}} F(t, x)\right|_{t=0}=2 k \sum_{n=0}^{\infty}(-1)^{n} q^{n h}(n+x)^{k-1},
$$

for $k \in \mathbb{N}$. Therefore, we obtain the following proposition.

Proposition 4.1. For $k \in \mathbb{N}$, one has

$$
\frac{G_{k, q}^{(h)}(x)}{k}=2 \sum_{n=0}^{\infty}(-1)^{n} q^{n h}(n+x)^{k-1} .
$$

From Proposition 4.1, we can derive the Genocchi zeta function which interpolates Genocchi polynomials related to $(h, q)$-Genocchi polynomials at negative integers.

For $s \in \mathbb{C}$, we define the Hurwitz-type Genocchi zeta functions related to $(h, q)$ Genocchi polynomials and numbers as follows.

Definition 4.2. For $s \in \mathbb{N}$, one has

$$
\zeta_{G}(s, x)=2 \sum_{n=0}^{\infty} \frac{(-1)^{n} q^{n h}}{(n+x)^{s}}, \quad \zeta_{G}(s)=2 \sum_{n=1}^{\infty} \frac{(-1)^{n} q^{n h}}{n^{s}}
$$

By Proposition 4.1 and Definition 4.2, we obtain the following theorem.

Theorem 4.3. For $k \in \mathbb{N}$, one has

$$
\zeta_{G}(1-k, x)=\frac{G_{k, q}^{(h)}(x)}{k}, \quad \zeta_{G}(1-k)=\frac{G_{k, q}^{(h)}}{k} .
$$

The generating function of the generalized $(h, q)$-Genocchi numbers attached to $X$ is given by

$$
F_{X}^{(h)}(t)=\sum_{a=0}^{d-1} \frac{2 t(-1)^{a} X(a) e^{a t} q^{h a}}{q^{h d} e^{d t}+1}=\sum_{n=0}^{\infty} G_{n, X, q}^{(h)} \frac{t^{n}}{n !},
$$

where $|h \log q+t|<\pi / d, d \in \mathbb{N}$ with $d \equiv 1(\bmod 2)$. Therefore, we have

$$
F_{X}^{(h)}(t)=2 t \sum_{n=0}^{\infty}(-1)^{n} X(n) q^{h n} e^{n t}
$$


where $x$ is a nontrivial character with conductor $d \in \mathbb{N}$ with $d \equiv 1(\bmod 2)$. From (4.8), it follows that

$$
G_{k, x, q}^{(h)}=\left.\frac{d^{k}}{d t^{k}} F_{X}^{(h)}(t)\right|_{t=0}=2 k \sum_{n=1}^{\infty}(-1)^{n} X(n) q^{h n} n^{k-1}
$$

This is equivalent to

$$
\frac{G_{k, x, q}^{(h)}}{k}=2 \sum_{n=1}^{\infty}(-1)^{n} X(n) q^{h n} n^{k-1} .
$$

For $d \in \mathbb{N}$ with $d \equiv 1(\bmod 2)$, let $\chi$ be a primitive Dirichlet character with conductor $d$. Then, we define

$$
L_{q}^{(h)}(s, X)=2 \sum_{n=1}^{\infty} \frac{(-1)^{n} X(n) q^{h n}}{n^{s}} \quad(s \in \mathbb{C}), h \in \mathbb{Z}
$$

From (4.10) and (4.11), we obtain the following theorem.

Theorem 4.4. For $k \in \mathbb{N}$, one has

$$
L_{q}^{(h)}(1-k, \chi)=\frac{G_{k, x, q}^{(h)}}{k} .
$$

\section{References}

[1] L. Carlitz, " $q$-Bernoulli numbers and polynomials," Duke Mathematical Journal, vol. 15, no. 4, pp. 987$1000,1948$.

[2] T. Kim, "Sums of powers of consecutive q-integers," Advanced Studies in Contemporary Mathematics, vol. 9, no. 1, pp. 15-18, 2004.

[3] T. Kim, "On the q-extension of Euler and Genocchi numbers," Journal of Mathematical Analysis and Applications, vol. 326, no. 2, pp. 1458-1465, 2007.

[4] T. Kim, "Note on q-Genocchi numbers and polynomials," Advanced Studies in Contemporary Mathematics, vol. 17, no. 1, pp. 9-15, 2008.

[5] T. Kim, L.-C. Jang, and H. K. Pak, "A note on $q$-Euler and Genocchi numbers," Proceedings of the Japan Academy. Series A, vol. 77, no. 8, pp. 139-141, 2001.

[6] S.-H. Rim, K. H. Park, and E. J. Moon, "On Genocchi numbers and polynomials," Abstract and Applied Analysis, vol. 2008, Article ID 898471, 7 pages, 2008.

[7] T. Kim, "Note on the Euler numbers and polynomials," Advanced Studies in Contemporary Mathematics, vol. 17, no. 2, pp. 131-136, 2008.

[8] I. N. Cangul, H. Ozden, and Y. Simsek, "Generating functions of the $(h, q)$ extension of twisted Euler polynomials and numbers," Acta Mathematica Hungarica, vol. 120, no. 3, pp. 281-299, 2008.

[9] L. Carlitz, " $q$-Bernoulli and Eulerian numbers," Transactions of the American Mathematical Society, vol. 76, pp. 332-350, 1954.

[10] M. Cenkci, "The $p$-adic generalized twisted $(h, q)$-Euler- $\ell$-function and its applications," Advanced Studies in Contemporary Mathematics, vol. 15, no. 1, pp. 37-47, 2007.

[11] M. Cenkci and M. Can, "Some results on $q$-analogue of the Lerch zeta function," Advanced Studies in Contemporary Mathematics, vol. 12, no. 2, pp. 213-223, 2006.

[12] M. Cenkci, M. Can, and V. Kurt, " $p$-adic interpolation functions and Kummer-type congruences for $q$-twisted and q-generalized twisted Euler numbers," Advanced Studies in Contemporary Mathematics, vol. 9, no. 2, pp. 203-216, 2004. 
[13] T. Kim, “On $p$-adic $q$-L-functions and sums of powers,” Discrete Mathematics, vol. 252, no. 1-3, pp. $179-187,2002$.

[14] T. Kim, "q-Volkenborn integration," Russian Journal of Mathematical Physics, vol. 9, no. 3, pp. 288-299, 2002.

[15] T. Kim, "On Euler-Barnes multiple zeta functions," Russian Journal of Mathematical Physics, vol. 10, no. 3, pp. 261-267, 2003.

[16] T. Kim, "Analytic continuation of multiple q-zeta functions and their values at negative integers," Russian Journal of Mathematical Physics, vol. 11, no. 1, pp. 71-76, 2004.

[17] T. Kim, "q-Riemann zeta function," International Journal of Mathematics and Mathematical Sciences, vol. 2004, no. 12, pp. 599-605, 2004.

[18] T. Kim, "Power series and asymptotic series associated with the $q$-analog of the two-variable $p$-adic L-function," Russian Journal of Mathematical Physics, vol. 12, no. 2, pp. 186-196, 2005.

[19] T. Kim, "A new approach to $p$-adic $q$-L-functions," Advanced Studies in Contemporary Mathematics, vol. 12, no. 1, pp. 61-72, 2006.

[20] T. Kim, "Multiple p-adic L-function," Russian Journal of Mathematical Physics, vol. 13, no. 2, pp. 151$157,2006$.

[21] T. Kim, "q-extension of the Euler formula and trigonometric functions," Russian Journal of Mathematical Physics, vol. 14, no. 3, pp. 275-278, 2007.

[22] T. Kim, "On $p$-adic interpolating function for $q$-Euler numbers and its derivatives," Journal of Mathematical Analysis and Applications, vol. 339, no. 1, pp. 598-608, 2008.

[23] T. Kim, "q-Bernoulli numbers and polynomials associated with Gaussian binomial coefficients," Russian Journal of Mathematical Physics, vol. 15, no. 1, pp. 51-57, 2008.

[24] T. Kim, "The modified $q$-Euler numbers and polynomials," Advanced Studies in Contemporary Mathematics, vol. 16, no. 2, pp. 161-170, 2008.

[25] T. Kim, "On the symmetries of the $q$-Bernoulli polynomials," Abstract and Applied Analysis, vol. 2008, Article ID 914367, 7 pages, 2008.

[26] T. Kim, "Symmetry $p$-adic invariant integral on $\mathbb{Z}_{p}$ for Bernoulli and Euler polynomials," Journal of Difference Equations and Applications, vol. 14, no. 12, pp. 1267-1277, 2008.

[27] T. Kim, "Euler numbers and polynomials associated with zeta functions," Abstract and Applied Analysis, vol. 2008, Article ID 581582, 11 pages, 2008.

[28] T. Kim, "A note on some formulae for the $q$-Euler numbers and polynomials," Proceedings of the Jangjeon Mathematical Society, vol. 9, no. 2, pp. 227-232, 2006.

[29] T. Kim, "A note on $q$-Volkenborn integration," Proceedings of the Jangjeon Mathematical Society, vol. 8, no. 1, pp. 13-17, 2005.

[30] T. Kim, "On the Sehee integral representation associated with $q$-Riemann zeta function," Proceedings of the Jangjeon Mathematical Society, vol. 7, no. 2, pp. 125-127, 2004.

[31] T. Kim, S.-H. Rim, and Y. Simsek, "A note on the alternating sums of powers of consecutive $q$ integers," Advanced Studies in Contemporary Mathematics, vol. 13, no. 2, pp. 159-164, 2006.

[32] T. Kim and Y. Simsek, "Analytic continuation of the multiple Daehee $q$-l-functions associated with Daehee numbers," Russian Journal of Mathematical Physics, vol. 15, no. 1, pp. 58-65, 2008.

[33] Y.-H. Kim, W. Kim, and L.-C. Jang, "On the q-extension of Apostol-Euler numbers and polynomials," Abstract and Applied Analysis, vol. 2008, Article ID 296159, 10 pages, 2008.

[34] L.-C. Jang, "Multiple twisted $q$-Euler numbers and polynomials associated with $p$-adic $q$-integrals," Advances in Difference Equations, vol. 2008, Article ID 738603, 11 pages, 2008.

[35] H. Ozden, I. N. Cangul, and Y. Simsek, "Multivariate interpolation functions of higher-order $q$-Euler numbers and their applications," Abstract and Applied Analysis, vol. 2008, Article ID 390857, 16 pages, 2008.

[36] H. Ozden and Y. Simsek, "A new extension of $q$-Euler numbers and polynomials related to their interpolation functions," Applied Mathematics Letters, vol. 21, no. 9, pp. 934-939, 2008.

[37] H. Ozden, Y. Simsek, and I. N. Cangul, "Euler polynomials associated with $p$-adic $q$-Euler measure," General Mathematics, vol. 15, no. 2, pp. 24-37, 2007.

[38] H. Ozden, Y. Simsek, S.-H. Rim, and I. N. Cangul, "A note on p-adic $q$-Euler measure," Advanced Studies in Contemporary Mathematics, vol. 14, no. 2, pp. 233-239, 2007.

[39] C. S. Ryoo, H. Song, and R. P. Agarwal, "On the roots of the $q$-analogue of Euler-Barnes' polynomials," Advanced Studies in Contemporary Mathematics, vol. 9, no. 2, pp. 153-163, 2004. 
[40] Y. Simsek, "Theorems on twisted L-function and twisted Bernoulli numbers," Advanced Studies in Contemporary Mathematics, vol. 11, no. 2, pp. 205-218, 2005.

[41] Y. Simsek, "On $p$-adic twisted $q$ - $L$-functions related to generalized twisted Bernoulli numbers," Russian Journal of Mathematical Physics, vol. 13, no. 3, pp. 340-348, 2006.

[42] Y. Simsek, "Generating functions of the twisted Bernoulli numbers and polynomials associated with their interpolation functions," Advanced Studies in Contemporary Mathematics, vol. 16, no. 2, pp. 251$278,2008$.

[43] Y. Simsek, O. Yurekli, and V. Kurt, "On interpolation functions of the twisted generalized FrobeniusEuler numbers," Advanced Studies in Contemporary Mathematics, vol. 15, no. 2, pp. 187-194, 2007.

[44] H. M. Srivastava, T. Kim, and Y. Simsek, "q-Bernoulli numbers and polynomials associated with multiple q-zeta functions and basic L-series," Russian Journal of Mathematical Physics, vol. 12, no. 2, pp. 241-268, 2005. 